\title{
Educational Psychology Theory Basis and Teaching Principles of Creative Music Teaching Method
}

\author{
Hong Xianli ${ }^{1, a^{*}}$, Chen Weijing ${ }^{2, b}$ \\ ${ }^{1}$ School of Education Science, Chongqing Normal University, Huxi, Shapingba, Chongqing, China \\ ${ }^{2}$ Shenzhen Songgang Orff Music Studio, Shenzhen city, Guangdong, China \\ a79322267@qq.com, bweijingalice@163.com \\ ${ }^{*}$ Corresponding Author
}

\begin{abstract}
Rich and diverse music activities can effectively develop special students' musical ability, improve their cognitive and motor coordination ability, and promote the harmonious development of their body and mind. Based on previous research and teaching practice, according to the Skinner's procedure teaching idea, Bruner's cognitive discovery theory, learning concept of the humanism psychology and constructivism, the author puts forward creative music teaching method in the field of special education. This paper mainly analyzes the educational psychology theory basis and teaching principle of the creative music teaching method.
\end{abstract}

Keywords: creative music teaching Method; educational psychology; teaching principles.

\author{
创造性音乐教学法的教育心理学理论基础及教学原则 \\ 洪显利 ${ }^{1,} a^{*}$, 陈玮静 ${ }^{2, b}$ \\ 1重庆师范大学教育科学学院, 虎溪, 沙坪坝, 重庆, 中国, 401331 \\ 2 深圳松岗奥尔夫音乐工作室，松岗，宝安区，深圳市，广东省，中国，518105

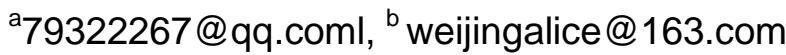 \\ *通讯作者
}

\begin{abstract}
中文摘要: 丰富多元的音乐活动能有效发展 特需学生的音乐能力、提高他们的认知和动 作协调能力，促进特需学生身心的和谐发 展。在前人研究和自身教育教学实践的基础 上, 根据斯金纳的程序教学思想、布鲁纳的 认知发现说、人本主义心理学和建构主义的 学习观, 作者提出了针对特殊教育领域的创 造性音乐教学法。文章主要分析了创造性音 乐教学法教学的教育心理学理论基础及其 教学原则。
\end{abstract}

关键词: 创造性音乐教学法; 教育心理学; 教学原则

\section{1. 引言}

\section{1 斯金纳的程序教学思想}

20 世纪 50 年代, 斯金纳完成了程序教 学的一系列研究。斯金纳认为, “教学就是 安排可能发生强化的事件促进学习”。他担 
心教师在课堂情境中不能提供适当而有系 统的强化, 因而不足以引起理想的学习效 果。所以他建议, 利用操作条件反射的理论 安排程序教学, 以更有效地呈现学习教材。 程序教学的目的在于通过使用机器装置来 提高学生在算术、阅读、拼写和其他学科的 学习效率, 希望机器能做某些胜过普通教师 所做的事情。这样可以使教师腾出时间, 从 事那些他能做得更好的工作。斯金纳程序教 学的基本思想是对学生的正确学习效果必 须给予及时的强化, 以鼓励学生继续进行学 习。程序教学的基本要求是：(1)小步子逻 辑序列。小步子逻辑序列就是把学习内容分 为许多具有内在联系的小单元, 编为程序, 每次只给一小步。最初的步子很容易, 逐渐 增加难度。教师要编写一系列刺激(问题) $\rightarrow$ 反应(答案)框面, 这些框面由易到难地小步 子地呈现教学内容。(2)要求学生必须主动 地学习。即要求他们对每个框面所呈现的内 容(问题)作出积极的反应。程序教材呈现给 学生的知识一般以问题的形式出现。这些问 题都要求学生通过填空、解题或书写答案作 出积极的反应。(3)及时反馈。给学生的每 个反应(答案)提供即时的反馈(指出正确答 案)。每一小步都附有正确的答案, 使学生 知道自己做得对不对, 这就是强化。强化越 及时越快, 效果越好。(4)低的错误率。尽 量安排好问题, 使学生能经常作出正确的反 应并得到及时强化。(5)学生自定步调。让 每个学生按照自己的进度完成整个教学程 序。(6)给勤奋和学习效果好的学生提供大 量支持性强化物。利用这种思想所进行的教 学称为程序教学。

这种程序教学的思想适合特需学生的 身心发展水平, 特别是智力落后学生。在创 造性音乐教学中, 我们对一首音乐的欣赏, 都是先从原位通过身体的动作进行音乐基 本节奏的感知, 然后再加入道具或乐器促进 对音乐节奏的感知, 循序渐进地逐渐增加学 习的要求, 在前一步基本达到要求后再进入 下一步的教学。

\section{2 布鲁纳的认知发现说}

美国杰出的教育心理学家布鲁纳认为, 学习是学习者以他原有的认知结构为基础, 主动地形成新的认知结构的认知过程。学生
不是被动的知识接受者, 而是积极的信息加 工者。人的认知过程正是主动地把进入感官 的事物进行选择、转换、储存和应用, 才得 以向环境学习并适应, 以致改造环境的。布 鲁纳很重视学生的学习过程, 认为重要的不 在于铭记多少事实, 而在于获取知识的过 程。布鲁纳认为, 学生的学习不仅要掌握学 科的基本结构, 还要掌握学习该学科的基本 方法, 其中发现的方法和发现的态度是最为 重要的。他认为, 教师对学习的最好的激发 乃是对于所学材料的兴趣, 即主要是来自学 习活动本身的内在动机, 而不是那些外部因 素, 诸如等级、奖赏、竞争等。使学生对学 习产生兴趣的最好途径就是使学习者主动 地 “卷入” 学习并从中体验到自己有能力来 应付外部世界。发现学习的方法也是激发学 生内部动机的一个有效途径。发现学习是以 培养探究性思维为目标, 以基本教材为内 容, 通过教师的引导、启发, 让学生从已知 的材料中概括出应有的原则和规律, 发挥聪 明才智, 从而获取新知识的学习。学生通过 发现学习, 不仅可以获得知识的体系(基本 结构)、发现的方法, 还能获得学习的自信 心和内部动机及发现和创造的态度。

由此可见, 发现的过程实质是一种体 验、感悟的过程。通过这种发现感悟, 有助 于学生获得知识的体系(基本结构)、学习方 法, 获得积极的学习情感和态度。在我们的 创造性音乐教学中, 我们通过设计一环扣一 环的教学体验活动, 促进学生在参与活动的 过程中, 感悟音乐的节奏与结构, 感悟人际 合作的美好, 感悟音乐的美好, 感悟自己创 新的成就感。

\section{3 人本主义心理学的学习观}

人本主义强调学习的基本原则必须是 尊重学生, 重视他们的意愿、情感和观念; 必须相信人能教育自己, 并认为学习就是个 人潜能的充分发展, 是人格的发展。其教育 目标指向学生个人的创造性、目的和意义, 是培养积极愉快、适应时代变化的心理健康 的人。人本主义的代表人物罗杰斯认为, 在 自我的发展过程中, 如果外界对学习者 “无 条件地关怀”, 那么他就会不断发挥其潜能, 并最终达到 “自我实现” ; 否则就会出现对 自我的威胁, 造成学习者的恐惧和适应不 
良。他认为, 教育的目标应该是促进变化和 学习, 培养能够适应变化和知道如何学习的 人。要实现这一教育目标, 必须采用意义学 习的方式。他所谓的意义学习, 不是指那种 仅仅涉及事实累积的学习, 而是指一种使个 体的行为、态度、个性以及在未来选择行动 方针时发生重大变化的学习; 不仅仅是指一 种增长知识的学习, 而且是一种与每个人各 部分经验都融合在一起的学习。这种学习包 括四个要素: 第一, 学习具有个人参与

（personal involvement）的性质, 即整个人 (包括情感和认知两个方面) 都投入学习活 动; 第二, 学习是自我发起(self-initiated)的, 即便推动力或刺激来自外界时, 但要求发 现、获得、掌握和领会的感觉是来自内部的; 第三, 学习是渗透性的 (pervasive), 即它 会使学生的行为、态度, 乃至个性都会发生 变化; 第四, 学习是由学生自我评价的

（evaluated by the learner）, 因为学生最清 楚这种学习是否满足自己的需要、是否有助 于导致他想要知道的东西、是否明了自己原 来不甚清楚的某些方法。因此, 他认为, 在 教学中, 教师应充分地尊重、信任学生, 为 学生创造一种真诚、关心、理解的气氛, 起 到 “助产士”与 “催化剂” 的作用。

因此, 我们认为, 创设问题情景, 引导 学生全身心投入学习, 感悟知识内在的含 义、联系、规律, 获得成功的体验, 有利于 促进学生真正的意义学习。在创造性音乐教 学中, 我们的活动从热身、发展到总结回顾 或安静活动, 从动作的模仿到自己的创意, 都是引导学生全身心参与的过程, 所以课后 学生的总结都很深入, 能从心底真正感受到 音乐本身的内涵和快乐。

\section{4建构主义的学习观}

建构主义心理学家认为, 学习是学习者 主动地建构内部心理表征的过程, 是学习者 原有的认知结构一一已经储存在长时记忆 中的事件和脑的信息加工策略, 与从环境中 接受的感觉信息 (信息) 相互作用, 主动的 选择信息和注意信息, 以及主动地建构信息 意义的生成过程。生成是学习中的基本认知 过程, 它是对学习材料中各部分关系以及学 习内容与知识、经验关系的积极建构; 学习 中的建构一方面是对新信息意义的建构, 同
时也包含对原有经验的改造和重组。信息意 义的生成过程就是一个“知识建构”的过程。 “知识建构”是学生从自身已有的知识和经 验出发, 对所要 “理解” 和 “消化” 的知识 作出自己的解释, 从而获得知识的意义。因 此, 学生获得知识的过程, 实际上是一种意 义发现的过程, 意义赋予的过程, 也是一种 意义创造的过程。“知识建构” 的过程是学 生的认知结构与认知策略、经验方式与情感 态度发生积极变化的过程, 也就是一种发展 和提高的过程。“知识建构” 的主体是学生, 教师是学生学习的辅导者和合作者。

在我们的创造性音乐教学中, 教师常常 会让学生感受音乐的基本节奏后, 画出自己 对音乐的感受, 然后再根据音乐结构进行小 组合作创编, 充分地发挥教师的引导和学生 的主动建构的作用。

\section{2. 特殊教育中的创造性音乐教学法的教学 原则}

\section{1创造性音乐教学设计课例}

为更好地说明我们的教学原则, 以一首 $\mathrm{AB}$ 结构的音乐欣赏的教学设计为例, 示范 我们的教学设计:

第一步, 基本节奏型 $(\times \times \times \times \times)$ 的感知: 示范+模仿、学生即兴三拍 (可声 音、可动作)

第二步, 原位动作模仿, 感知音乐结 构: $\mathrm{A}$ 拍腿 2 次, 拍手或肩或头 3 次; $\mathrm{B}$ 摇手 7次, 空中双手上举停 1 下; 或拍自己身体7 次, 空中双手上举停1次; 或双手互转7次, 空中双手上举停一次。

第三步，两人对坐（方式一）：A拍腿2 次, (朋友)握手或拍手或拉钩 3 次; B手拉扯 7次，空中对拍 1 下。

两人对坐 (方式二)：A拍腿2次, （亲 子）拥抱或贴脸或点额头 3 次; $\mathrm{B}$ 与对方即 兴互动身体。

第四步, 两人组 (原位, 大圆圈) : A 踏步2次、与对方用身体打招呼 (肩、屁股、 膝盖）3次； $\mathrm{B}$ 与朋友拉扯跳。

第五步, 内外两圈: A内圈不动, 做声 势（踏步2次、与对方握手或拍手或拉钩 3 次）; 外圈移动 2 步换舞伴, 拍对方手 3 次; $\mathrm{B}$ 与新舞伴自由跳。 
第六步, 两人听音乐自由行走: $\mathrm{A}$ 手搭 手自由行走 2 步、拍对方手 3 次; $\mathrm{B}$ 与朋友挽 手臂8拍, 然后换伴3次。

第七步，原位坐： $\mathrm{A}$ 音筒坚起摇2次、 用音筒敲地或腿或肩 3 次; $\mathrm{B}$ 地上用脚滚动 音筒或用手玩或按摩身体

第八步, 内外两圈: A 踏步2次、敲对方 的音筒 3 次, 然后换伴; $\mathrm{B}$ 以音筒连接 2 人自 由跳

第九步, 内外两圈: A内圈不动, 做声 势（踏步2次、与对方敲音筒3次）; 外圈移 动2步换舞伴, 拍对方手 3 次; $\mathrm{B}$ 手握音筒 连接右、左、前、后各跑8拍。

第十步, 原位坐用打击乐器感知基本 节奏型。

第十一步, 为音乐配器: $A$ 拍鼓或刮胡 2次、打棒或双响筒3次; B沙锤或铃鼓及丝 巾。

第十二步，移位： A 拍鼓或刮胡2次、 敲打棒或双响筒3次; B沙锤或铃鼓或丝巾 自由跳（分 $\mathrm{AB}$ 两组, 一组动, 一组定格)。

\section{2 特殊教育中的创造性音乐教学法教学 的教学原则}

教育不是功利的事情, 是帮助特需学生 探索自我成长的一个过程。并不是特需学生 不懂、不会欣赏经典, 重要的是你怎么引领 他进入艺术之门。如果你用很简单、快乐的 游戏引导, 学生不知不觉䢟游在艺术的海洋 里。重要的是教师的真诚和宽容, 聚焦过程 的人性化, 支持特需学生健康人格的发展。 创造性音乐教学中, 教师能掌握各种材料, 释放孩子潜在的自然能量。上述课例只是以 一首结构简单的音乐为例展示了我们在特 殊教育实践中的创造性音乐法的基本教学 过程及方法。在我们针对特殊学生设计的创 造性音乐教学中, 除了常常使用结构简单的 音乐外, 我们还常使用某一种简单的乐器、 简单的嗓音、生活动作、单一的生活元素或 主题为中心开展一系列合理设计, 稳妥、扎 实地导向创作音乐时的各种元素的敏锐探 索, 循序渐进地帮助孩子成长。概括起来, 所有的创造性音乐教学活动, 教师都需要遵 循以下原则:

第一，小目标、小步子教学，前一个目 标没实现时不能进入下一个更高的目标。由
于特需学生的接受能力比较慢, 对学生的教 学必须分解为小步子, 一次一个小要求地渐 进教给学生。在一小步一小步的重复和渐进 学习中, 特需学生获得熟悉感和胜任感, 获 得学习的乐趣。同时, 教学环节之间环环相 扣, 层层递进, 一次增加一个新的元素, 逐 个变换活动; 增加的元素可以在A段, 也可 在B段; 先示范再模仿。

第二, 遵循种子教学原理, 以音乐种子 为中心, 设计活动多元变化、万变不离其宗。 音乐 “就像一粒种子, 播种培育就可能长成 一棵参天大树, 而这种发展 (活动形式) 可 以是各种各样的。”教师不是用语言解释概 念而是在音乐中体验。遵循布鲁纳的动作表 征到映象表征再到符号表征的认知发展三 阶段理论, 用好身体乐器本身, 活动设计先 让学生的身体充分感受音乐的结构与节奏, 再加入道具, 乐器最后进入; 活动形式由个 体到群体, 由 1 人到 2 人、 4 人、群体; 活 动由原位坐、站到移位的行走、舞蹈; 动静 结合; 每种活动应让学生积极参与, 活动多 元变化、万变不离其宗。多样化的形式, 刺 激特需学生的听觉、触觉、本体觉、动觉、 视觉的多感官从不同角度感受音乐, 形成对 音乐的整体感知和协同, 促进身心的协调。

第三, 活动设计要突出音乐的节奏和特 性, 动作、乐器与音乐特性融为一体。围绕 简单的曲式结构, 把语言、声势、动作、歌 唱和乐器等巧妙地编织在一起。这些结构反 过来创设美好、安全的氛围让特殊孩子全身 心、自由自在地参与进来, 螺旋式建构发展, 无须把追求成功所带来的压力看得过重, 有 智慧地自然面对, 应确保每一水平上直接获 得其音乐成就。

第四, 教师引导与学生主体的结合, 留 有学生个性和创造性发挥的空间。所有课程 都遵循一个基础性的连续统一体: 从 “教师 的模仿” 到 “学生的创造”。在模仿与创 造中, 音乐魔法箱中永远涌现不断的惊喜。 有时温故也能知新, 也会比以前做得更好或 更新, 在平凡的生活中发现新的创意和想 法, 这种能力亦称作创造力。教师应用学生 的方式去尝试和修正, 而不是用教师自己的 方式强加给学生, 教与学是互相学习和吸收 的相生过程。犹如空杯, 越开放能量越多。 
注重因材施教, 根据学生能力要求调整适宜 的活动, 不断寻找新的途径, 提供大量学生 创作和即兴的机会。在给学生开放的过程, 学生之间的合作与创造力得以充分地发挥, 教师的教学方法也得到新的拓展, 从而真正 体现创造性音乐教学法的创造性。

第五, 教学基本流程: 热身-引入主题多样化练习-小结和记录时间。每次教学都 要有热身活动, 打开身体, 联系彼此, 建立 安全的人际环境。多样化地练习可以是唱歌 和声乐活动、声势和乐器演奏、舞蹈和律动、 倾听和欣赏、音乐记录和音乐写作, 以多样 化练习刺激学生的不同感官对同一信息的 接收, 促进学生对音乐的理解和建构, 获得 音乐的快乐。每次教学都要留有回顾与总结 的时间, 引导学生去回顾整个过程, 提炼自 身对音乐的感悟。

第六, 精心准备, 收放自如。每个活动 都有目标和结构, 教师对于每一个步骤必须 了然于心。每个活动应能引起学生的兴趣, 通常以游戏引入, 学生在循序渐进的游戏式 学习中成长音乐素养及人格素养。花大量的 时间准备是值得的, 只有做好充分准备, 才 有强大的信念, 才会感染课堂上的每一个学 生。教师应能对活动收放自如, 搜寻和发展 适合学生人格发展的材料并组织教学, 引导 学生健康人格的发展。所有的活动应适合每 一个学生, 对活动应有责任感, 做到对音乐 负责、对学生负责、对社会负责。

\section{3. 结束语}

根据国家颁布的全日制培智学校课程 计划, 其中的 “唱游与律动、艺术休闲” 属 于音乐教学的领域。特殊需求学生指学龄期 学生在认知、语言、肢体动作、社交或情绪 等方面出现任何一种或一种以上发展迟缓 情形者, 简称特需学生。特需学生由于肢体 或情绪或认知等单一或多方面的障碍, 导致 其学习和发展受到诸多的限制。怎样让我们 的音乐教学更好地适应孩子的特点, 并促进
其发展, 成为音乐教师探讨的重要课题。在 多年的实践中, 我们发现通过丰富多元的音 乐活动能有效发展特需学生的音乐能力、提 高他们的认知和动作协调能力, 促进特需学 生身心的和谐发展。柔美的音乐氛围能够让 特需学生感受到音乐的美好力量, 从而提升 学生的生活品质。

为此, 我们在前人研究和自身教育教学 实践的基础上, 根据相关教育心理学学习理 论, 提出了针对特殊教育领域的创造性音乐 教学法。创造性音乐教学是指根据特需学生 身体、心理的需求, 利用多种艺术形式如韵 文、歌唱、律动、绘本、乐器演奏、戏剧等 艺术活动, 促进特需学生人格发展的丰富及 圆满。特教领域创造性音乐教学的理念、方 法综合了奥尔夫音乐教学体系、融合创意舞 蹈、形体戏剧、融合创意音乐剧等, 目标是 用音乐、美术、舞蹈、戏剧的方法促进特需 学生身心发展并提升其生活品质。本文将主 要分析特殊教育领域中创造性音乐教学法 教学的教育心理学理论基础及其教学原则。

\section{References}

[1] Hong XianLi etc. The classical theory of education psychology and its application [M].Beijing university press, pp. 60 ,2011

[2] Zhang Chengfen. Education psychology $[\mathrm{M}]$. Shandong education press, pp. 78,2000 .

[3] Shi Liangfang, The Learning theory The learning psychology theory and principle $[\mathrm{M}]$. People's education press, pp. 405, 1994.

[4] Hong XianLi etc. The classical theory of education psychology and its application [M].Beijing university press, pp. 27,2011

[5] Li Dana, Xiu Hailin, Orff music education thought and practice $[\mathrm{M}]$. Shanghai education press, pp. 62,2011. 